\title{
Methods of Temperature Monitoring in Low Voltage Electrical Cables using Composite Materials
}

\author{
Mircea Florin Drăghici ${ }^{1}$, and Maria Stoicănescu ${ }^{1, *}$ \\ ${ }^{1}$ University Transylvania University of Brasov, Department of Materials Science, Brasov, Romania,
}

\begin{abstract}
The article presents the most important methods and technologies used to monitor the temperature of low voltage power supply cables, which supply $400 \mathrm{~V}$ in three-phase mode, trying to highlight the method of using composite materials, materials that are included in the material constructive of the cables, these having the role of temperature sensor. Temperature monitoring is performed along the electrical cables, to transmit in real time information on the evolution of temperatures along the entire length of the cable, at any point, during their entire life. The article will focus on the method of inserting optical fibres inside the power supply cables, which will be used as a temperature measuring instrument. Using the Reman effect, a method of reflecting the injected light at one end of the optical fibre, by processing the received signals, the evolution of the temperature inside the electric cable can be detected with great precision, over long distances and with great accuracy. the evolution of the temperature inside the electric.
\end{abstract}

\section{Introduction}

For the distribution of electricity in the national electricity system, a special role is played by the electricity transformation stations and sub distribution stations connected to the electrical supplier network, but also those owned by the end user [1].

At present, most of the transformation stations and sub-distribution stations, but also those of electricity transmission to these stations, being built many years ago, have outdated equipment, with advanced moral and physical wear, which will involve their replacement during next period [1].

The impact of the aging of electricity distribution facilities is related to the reliability and operational safety offered to the electricity consumer [1].

To have continuity in the power supply of an electrical receiver, the correct and safe operation of the power supply circuit will be considered.

One of the most important components of the power supply circuit is the electrical cables that will be discussed in this article.

To know the technical condition of the electric cables, it is necessary to monitor some functional parameters, respectively their diagnosis.

\footnotetext{
*Corresponding author: stoican.m@unitbv.ro
} 
Temperature is especially important during the operation of a power supply cable, most often the overheating of the power cable having a decisive role in its damage, respectively in the interruption of the power supply circuit.

In an electric cable, under voltage, heat is constantly developed, due to the transformation of part of the electromagnetic energy transported into thermal energy.

Therefor a spontaneous heat flow occurs because of the heat developed inside the electric cable. That flow migrates from the highest temperature area of the cable to the lowest temperature area.

Monitoring the evolution of temperature in electrical cables can be done occasionally or continuously.

Currently, in the electrical energy distribution installations, the monitoring is done using the continuous mode at the transformers, and at the electric transport cables, at the electrical switching devices and the connection equipment, an occasional monitoring is done [2].

Excessive heating of the power supply cable endangers its proper operation, shortens its life, bringing changes in electrical parameters (voltage losses along the length of the cable) to the power supply.

A possible occurrence of possible malfunctions due to thermal stresses in a production unit can lead to loss of profit, inability to achieve the proposed objectives, continuous monitoring of the temperature of power cables for electrical receivers used in the production of the unit contributing to an improvement management of electrical equipment of the unit.

To increase the operational safety of the electrical installations and to increase the life of the power supply circuit, the present paper proposes to make a continuous temperature monitoring for the low voltage power supply cables.

\section{Methods for monitoring thermal stresses for electrical cables}

Using different methods of temperature monitoring inside an electric cable, temperature variations along the cable can be quickly identified, and it can be easily seen if these temperature values meet the permissible limits, thus preventing and reducing negative effects. (technical and economic) of any damage that may occur, directly in the monitored cable or in the receiver to which the power supply is provided [3].

Using continuous temperature monitoring equipment, the information can be processed by certain devices, which can generate alarms, information storage, remote information transmission via a communication device.

For a homogeneous conductor of length $l$ and constant cross-section $s$, assuming a constant value of the current density, the power dissipated as heat in the unit of time is given by the relation.

$$
P=\int_{Y} \rho J^{2} d V=R i^{2}
$$

$R$ being the resistance of the conductor, and $i$ the intensity of the current passing through it.

Thermal transmission is achieved in known ways: conduction, radiation and convection, the highest speed in metals, the rarefied gases being characterized by the weakest thermal conduction.

The heat exchange between bodies with different temperatures, carried out by radiant energy, is the thermal transmission by radiation. This usually takes place in transparent environments for infrared and light radiation, with wavelengths in the range $0.8 \div 40 \mu \mathrm{m}$, respectively $0.4 \div 0.8 \mu \mathrm{m}[3]$.

For the transient heating regime of a current path by Joule-Lenz transformation represents the effect of the changes that occur, usually by jump, in the values of the current intensity that crosses the conductor; during any transient regime, temperatures are variable over time. 
Transient thermal regimes of conductors occur both during normal operating regimes of installations, when they are subjected to long-term thermal stresses (continuous or intermittent), and during abnormal (short-circuit) regimes, when the thermal stress is short duration [3].

The study of the transient heating regime of a conductive path in case of long-term thermal stress is performed based on the following simplifying hypotheses:

- the conductive path is homogeneous,

- the global thermal transmittance and specific heat are considered invariant with temperature,

- the temperature variation along the conductor is zero, and the ambient temperature has a constant value [3].

The heating regime of the conductive paths corresponding to the short-term thermal stress, produced under the action of short-circuit currents, is called thermal stability [3].

The thermal stability of electrical conductors / cables represents their ability to withstand, for a limited time and without visible damage, the thermal stress produced by short-circuit currents [9].

The mentioned durations and the corresponding current intensity have values specified in the norms and constitute nominal parameters of the electrical equipment.

Monitoring the temperature of electrical conductors / cables can be one of the best methods of predictive cable maintenance [4].

At present, the thermal monitoring of electrical cables is performed with monitoring devices of two types:

-In direct contact with the cable / conductor,

- Devices using non-electrical sensors are based on the expansion of a material, information that can be taken over by a certain type of displacement transducer,

- Devices using electrical sensors - thermocouples, p-n junctions, acoustic waves,

-Without direct contact with the cable / conductor - here being the quantum detectors infrared thermal imaging cameras, etc.

Temperature monitoring can be performed in several ways based on different phenomena and physical effects, in which the change in temperature produces changes in some properties or characteristics of materials, such as:

- $\quad$ variation in volume (by thermometers);

- $\quad$ variation of electrical resistance (by thermoresistors);

- electromotive voltage between the junctions of two different metals (by thermocouples);

- $\quad$ variation of the emitted radiation intensity (by thermographic camera);

- variation of the resonant frequency of a piezoelectric material.

Monitoring the temperature of electrical cables / conductors either under the insulating sheath of the electrical cable / conductor or on the surface of its sheath will bring high precision information to the user, information that can be processed, stored using automatic devices, based on algorithms programmed and installed in these [5].

\subsection{Infrared thermographic monitoring method}

Represents some of the most modern methods of temperature monitoring, being used in maintenance times programmed by the user, having the advantages that: it has a short time to obtain measured values, there is no physical contact between the measuring device and the tested cable surface, does not require preparation of the tested surface.

The method has the disadvantage that it identifies the possible abnormal temperature values of the analysed cables only at the measurement moments, being an occasional monitoring mode [6]. 
Other disadvantages would be that the measured temperature values are influenced by solar radiation, shade, humidity [6].

Having a thermal imaging camera, you can see in the figure below (Fig. 1) the temperature demands to which the low voltage electrical cables are subjected, which supply electrical receivers from a production unit.

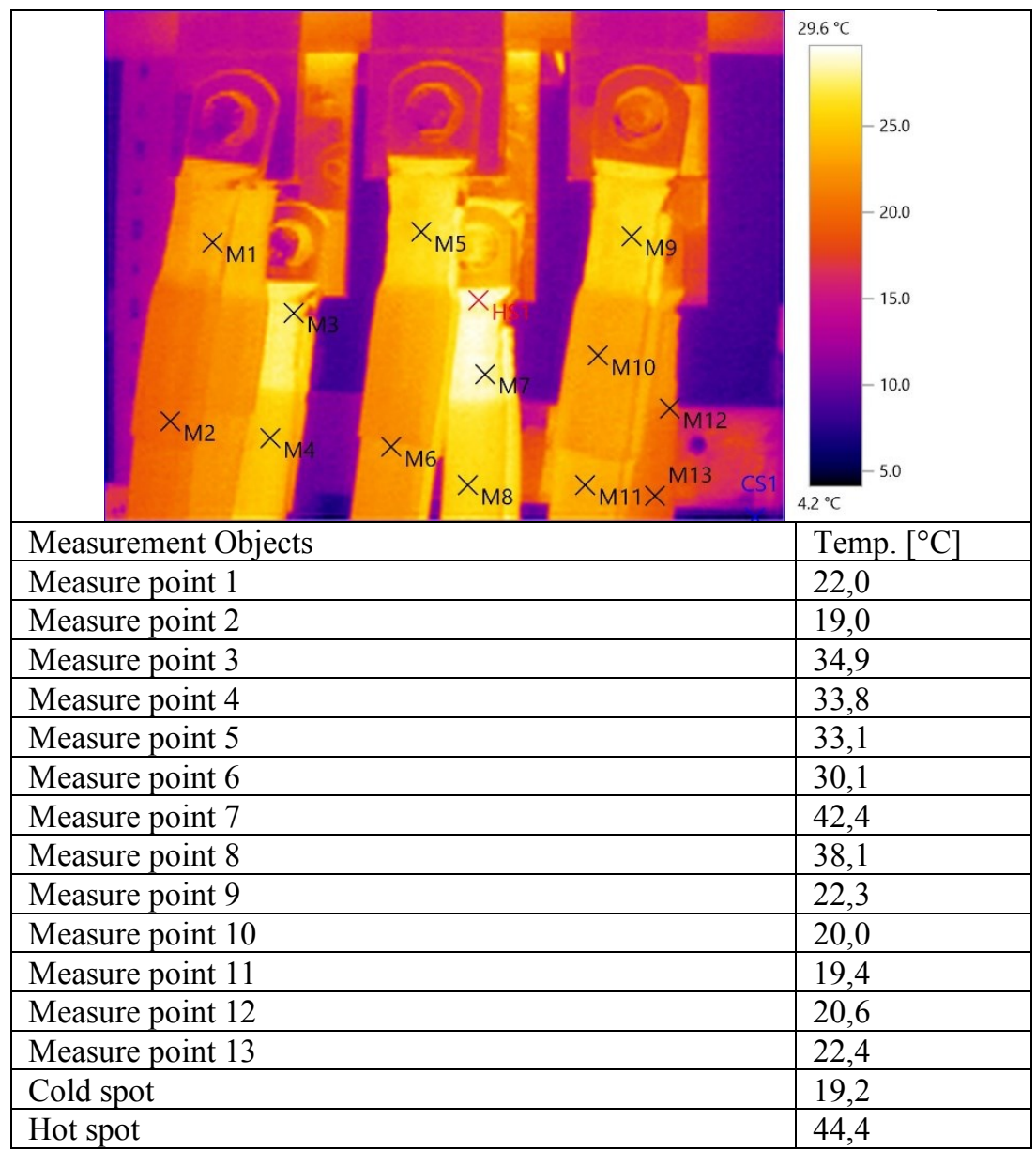

Fig. 1. Monitoring the cable temperatures with the help of the thermal imaging camera. The image was recorded in an existing industrial production factory, on an electrical supply power circuit.

\subsection{Finite Element Model (FEM)}

The finite element method allows the analysis of physical phenomena, such as temperature measurement, which can be described using mathematical models consisting of systems of differential equations with initial and extreme conditions.

The simple equations that model finite elements are then assembled into a larger system of equations that model the whole problem. (FEM) uses variational methods in calculating variations to approximate a solution by minimizing an associated error function [7].

A finite element model (FEM) method was used to calculate the dynamic rating in [7]. However, both methods require temperature measurements to initialize the TEE thermal 
model. This requires one or more temperature detection measurements installed along the cable path.

Thermal analysis of power cables is important for the determination of current carrying capacity. Cable temperature depends on current following through the cable, cable structure, material properties, laying form and thermal properties of environment of the cable.

For one XLPE cable, the effect of buried depth on heat distribution has been investigated; it is observed that current-carrying capacity of cables increased when laid near the surface [8].

\subsection{Node voltage method}

The node voltage method is one of the most used methods for visualizing problems at the electrical circuit level.

This method is a mathematical calculation method because the thermal circuit of an electric cable is equivalent to the electric circuit of the electric cable.

By measuring in real time, the temperature of the polymeric coating of the cable, the temperature of the conductor inside the cable can be determined by calculation, using the node voltage method $[9,13]$.

\subsection{Cable temperature monitoring based on the Phasor Measurement Units (PMU) method}

With the current evolution of technology, online monitoring of voltage stability through measurements made over time by phasor measuring devices seems possible [10].

The parameters of the monitored cable (electrical resistance, reactance, susceptibility) can be estimated based on the method of measuring the voltage phasor [10].

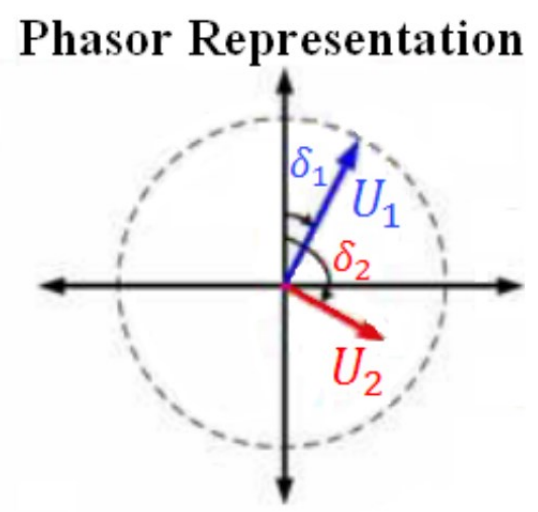

Fig. 2. - Phasor representation

The advantage of this cable temperature monitoring method is that it does not require additional temperature sensors located along the cable, the method being based on a mathematical algorithm, which provides accurate values of electrical resistance for various segments of three-phase cables.

The results from the network data demonstrate that the method has a temperature monitoring accuracy of $\pm 5^{\circ} \mathrm{C}[10]$. 


\subsection{Cable temperature monitoring method using optical fibre -DTS (Distributed Temperature Sensing)}

Distributed temperature detection (DTS) systems are optoelectronic devices that measure temperatures through optical fibres that act as linear sensors.

DTS systems contain a pulsed laser that sends a pulse of about $1 \mathrm{~m}$ (equivalent to a time of $10 \mathrm{~ns}$ ) in the optical fibre. As the pulse moves along the length of the optical fibre, it interacts with the glass. Due to the small imperfections in the glass, a small amount of the original laser pulse is reflected back to the DTS detection system [11,12].

By analysing reflected light, DTS is able to calculate the temperature of the event (by analysing the power of reflected light) and also the location of the event (by measuring the time it takes for the diffused retro light to return) to typically one meter $[11,12]$.

Temperatures are recorded along the optical sensor cable, so not in points, but as a continuous profile.

The determination of the temperature (with Reman effect inside fibre) along the optical fibre, in long distances has precise results.

An optical laser pulse sent through the fibre results in a scattered light that is reflected back to the transmission end, where the information is analysed [11].

The intensity of Raman scattering is a measure of temperature along the fibre. The antiStokes Raman signal changes its amplitude significantly as the temperature changes; the Raman Stokes signal is relatively stable.

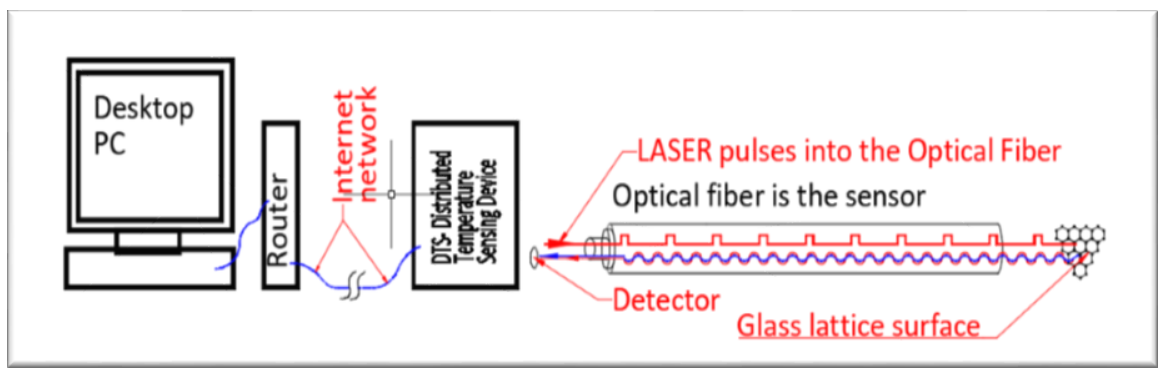

Fig. 3. - Representation of the system that analyse the distribution of the temperature along the optical fibre, named DTS

DTS technology analyse Optical Frequency Field Reflectometry (OTDR or Raman OFDR) discovered by the Indian physicist Sir Chandrasekhara Venkata Raman (1888-1970), According to his study the light passes through a transparent material, some of the deflected light changes in the wavelength. This innovative work in the field of light scattering brought him the Nobel Prize in Physics [14].

Due to the time of the return light pulse we can determine accurately the temperature along the optical fibre.

The coding allows the use of low optical power, eliminates any problems related to laser degradation and allows long-term measurement stability.

Among the uses of DTS technology is the monitoring of the temperature of electrical cables [15].

One of the known applications are those in which DTS technology is used to monitor the temperature of medium voltage cables $(110-220 \mathrm{kV})$ [11,12].

This application monitors the maximum temperature allowed at the surface between the conductor and the cable insulation $\left(90^{\circ} \mathrm{C}\right.$ for the $220 \mathrm{kV}$ cable between Siems-Luebeck localities) [16]. 

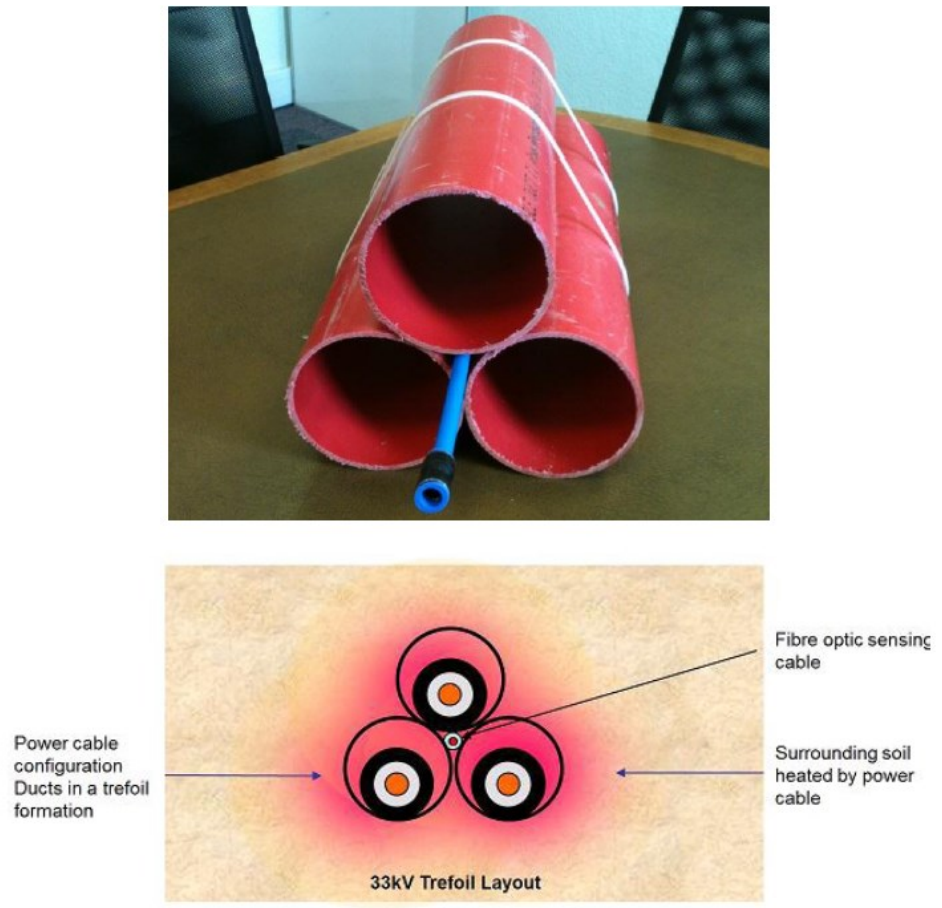

Fig. 4 Represents the arrangement of PVC protective pipes, electrical cables and fibre optic cable (OFC) in a $220 \mathrm{kV}$ circuit [16].

Using real-time temperature assessment (RTTR) software, the temperature along the medium voltage electrical cable is monitored [16].

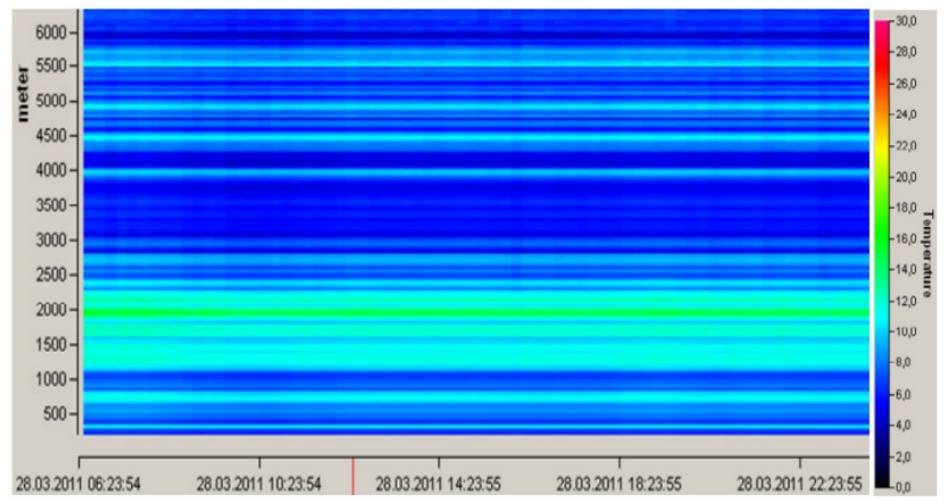

Fig. 5 Detailed visualization of the temperature changes along the medium voltage cable using a dedicated software [16] (VISCOM)

The VISCOM Client software calculates the temperature along the cable, providing information from a long period of time stored in the internal memory of the terminal device. 

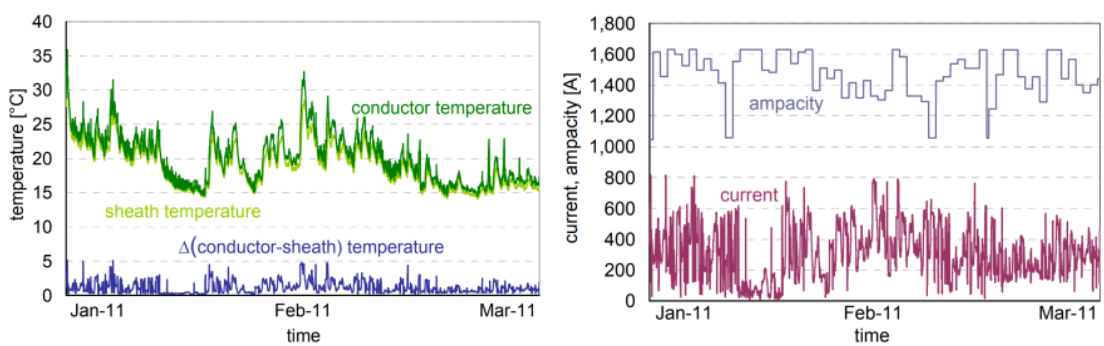

Fig. 6. The evolution of the temperature of the conductor and its sheath over time, respectively the current load of the cable during that monitored period [16].

\section{Conclusion}

The main purpose of the article is to prove the importance of obtaining information about the inner temperature inside the low voltage electrical cables, this is done using the DTS system, and the most suitable place for mounting the fibre optic cable for temperature detection is inside the cable, not on its outside as it is practiced in medium and high voltage cable systems.

The optical fibre is a glass or composite fibre (plastic) that has the property of transporting light along it. Optical fibres are widely used in the field of telecommunications, as they have the property of allowing the transmission of the signal over very long lengths with very small signal losses, also the transported signal is not influenced by electromagnetic interference of areas where these fibres are installed.

For the low voltage three-phase multi-wire cables $(400 \mathrm{~V})$, the ideal place to install the optical fibre is in the middle of the cable, between the three-phase cables, this optical fibre being protected by a coating resistant to mechanical challenges due to cable movements during transport and installation final, the respective coating having a high thermal conductivity.

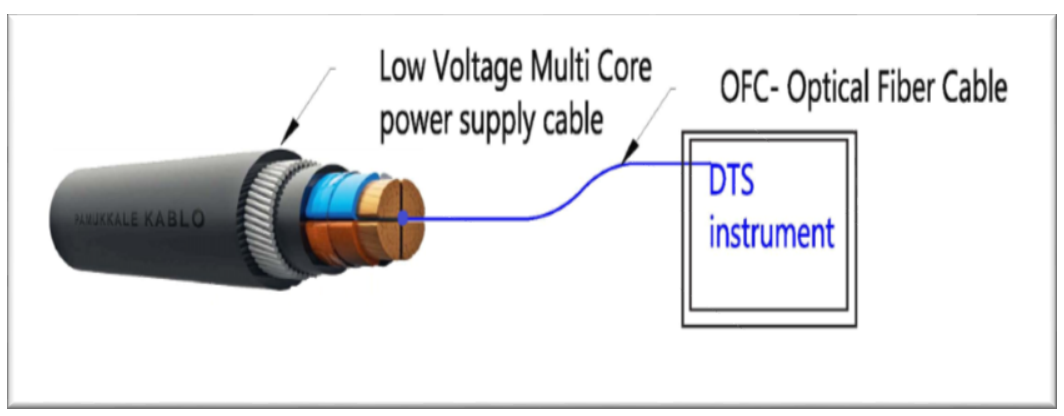

Fig. 7. Proposal for mounting the optical fibre in the low voltage multi-wire cables

My proposal for low-voltage, single-phase fibre optic temperature sensing electrical conductors can be mounted in the insulation sheath of the conductor, in a sheath similar to that described for low-voltage multi-wire cables. 


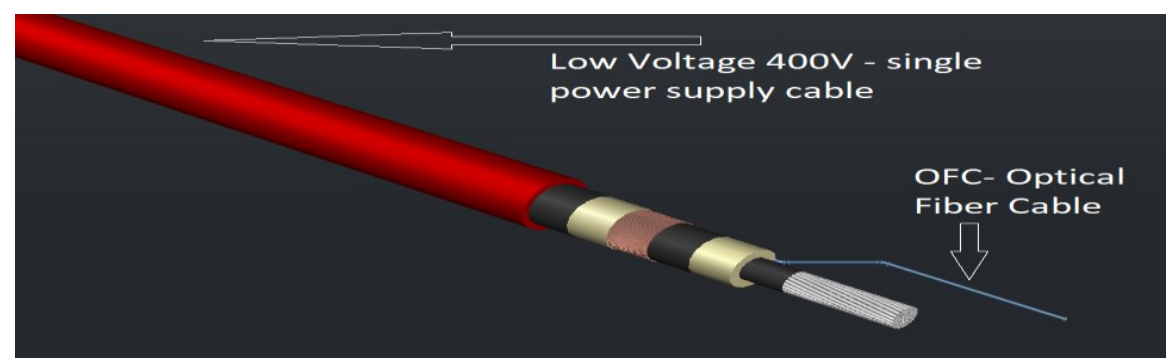

Fig. 8. Proposal for mounting the optical fibre in the low voltage conductors

With practical research we can determine the smallest section of the cables can accept to be installed inside of them an optical fibre for temperature detection.

The temperature along a low voltage electrical cable are not constant values, this parameter is variable and depending on the environmental conditions in which the electrical cable is installed (ground mounting, ground mounting depth, air mounting, ambient temperature, etc.)

When the cable is connected to the terminals and is starting the commissioning, the cable temperature monitoring system with DTS system, all these environmental values should be known, respectively entered in the monitoring software system as calculation input parameters.

One of the main advantages of monitoring the main power distribution cables in a low voltage installation is the complete thermal visibility along the monitored cables, respectively receiving information about the fact that those cables do not have an electrical overload, providing o safety in the operation of those installations.

The normal changing of the electric charge of the cables over time, (without exceeding the nominal charge indicated by the electric cable manufacturer) does not bring major changes in the temperature of the cable.

The optimal solution for the new objectives in which new electrical installations are executed is to deliver low voltage electrical cables, for the main circuits, having installed, directly by the electric cable manufacturer, inside them optical fibre for temperature detection.

At the terminal ends of the electrical cables, where the fibre optic detection terminal installed inside the cable is located, a device for connecting the optical fibre and processing information on the temperature along the cable will be mounted.

If the OFC terminal device is equipped with a communication modem and internal memory, it can be connected in a data transmission network, this information can be collected and processed remotely.

The installation of optical fibres for detecting the temperature values inside the electrical cables can ensure them a long life, not being affected by the influence of the external environment.

Increasing the number of manufacturers of low voltage electrical cables with fibre optics built into them would have a significant impact in reducing accidents due to overloading of electrical circuits.

One of the disadvantages of optical fibres is that the connection to the end ends requires complex technologies, the end ends must be cut carefully, the connection to the end devices is made either by mechanical connection or by welding the ends. Specially dedicated connectors are used to connect the end ends of the fibres.

At present, the prices for optical fibres, end connectors, manufacturing technology have dropped a lot. Optical temperature detection fibres inserted in low voltage cables which are supplying the power receivers, to monitor the evolution of temperatures can be used mainly 
in hospitals, industrial production units, automotive production industry, etc. increasing the operational safety of these buildings.

\section{References}

1. A. A. Adăscăliţei, Teoria circuitelor electrice (Editura Performantica, Iași, 2015)

2. E. Ivan, A. G. Husu, Materiale utilizate în ingineria electrică, (Editura Bibliotheca, Târgoviște, 2009)

3. IEC 60287-2-1:2015, Electric cables - Calculation of the current rating - Part 2-1: Thermal resistance - Calculation of the thermal resistance (2015)

4. G. Mazzanti, IEEE Transactions on Dielectrics and Electrical Insulation, 16, (2009)

5. E. C. R. Bascom, 2014 IEEE PES T\&D Conference and Exposition, (Chicago, IL, USA, 2014)

6. M. Hohmann, Electrical Construction and Maintenance, 98, 9 (1999)

7. M.A. Gray, IEEE Computing in Science \& Engineering, 10, (2008)

8. J. Luo, Y. Liu, Proceedings Electrical Insulation Conference and Electrical Manufacturing Expo, (Indianapolis, USA, 2005)

9. D. Enescu, P. Colella and A. Russo, Energies, 13, 5319, 13, 20 (2020)

10. D. Shi, D. J. Tylavsky, European Transactions on Electrical Power, 21, (2011)

11. A. Ukil, H. Braendle, P. Krippner, IEEE Sensors Journal, 12(5),885 (2012)

12. M. Schmale, H. Dräger, Cigré Session 2010, B1, 113, (2010)

13. R. J. Millar, A comprehensive approach to real time power cable temperature prediction and rating in thermally unstable environments, TKK Dissertations 46; Espoo 2006; Doctoral Dissertation [Online]. Available: www.researchgate.net/publication/27516483 [Accessed 10.04.2021]

14. https://ro.wikipedia.org/wiki/Chandrasekhara_Venkata_Raman [Accessed 10.04.2021]

15. M. Ohashi, Optics Express, 29, (2021)

16. Geoff Murphy, Temperature monitoring windfarm cable circuits Tier 1 [Online]. Available:https://www.ofgem.gov.uk/sites/default/files/docs/2015/08/temp monitoring _windfarm_cable_circuits final_1.pdf,(2015) [Accessed 10.04.2021] 